\title{
Coastal trapped disturbances en el litoral catalán: un peligro aeronáutico
}

https://doi.org/10.31978/639-19-010-0.241

\author{
Sergi González Herrero ${ }^{1,2}$ (sgonzalezh@aemet.es) \\ Alfons Callado Pallarés ${ }^{1}$ (acalladop@aemet.es) \\ Ernest Werner Hidalgo ${ }^{1}$ (ewernerh@aemet.es) \\ Pau Escribà Ayerbe ${ }^{1}$ (pescribaa@aemet.es) \\ Joan Bech Rustullet ${ }^{2}$ (joan.bech@meteo.ub.edu)
}

${ }^{1}$ AEMET / Delegación Territorial en Cataluña

${ }^{2}$ Universitat de Barcelona / Departamento de Física Aplicada - Meteorología

\begin{abstract}
RESUMEN
Un promedio de cuatro veces al año en el aeropuerto de Barcelona se produce un fenómeno local que constituye uno de los mayores peligros en las operaciones aéreas dentro del Estado español. Este fenómeno consiste en un incremento de la velocidad y cambio de dirección bruscos del viento, junto con un aumento temporal de la cizalladura vertical y horizontal. Este fenómeno ha sido descrito como coastal trapped disturbances (CTD). En esta comunicación se describe el modelo conceptual de este fenómeno en el Mediterráneo noroccidental mediante el uso de modelos de alta resolución (HARMONIE-AROME) centrándose en las consecuencias que produce en la operatividad aeronáutica y proponiendo indicios conceptuales para incrementar su predicción.

Las CTD están asociadas comúnmente a las situaciones de tramontana-cierzo. Climatológicamente se producen durante la mitad cálida del año y por la tarde. Las CTD se desarrollan cuando una masa de aire frío al norte de Barcelona avanza como una corriente de densidad hacia el suroeste. $\mathrm{Al}$ avanzar, la fuerza de Coriolis desvía ligeramente la masa de aire hacia la cordillera costerocatalana. La interacción del aire con las montañas genera un forzamiento que actúa como acelerador del viento a la vez que genera una fuerte cizalladura en la frontera entre masas de aire. Finalmente, las CTD mediterráneas se desarrollan a escalas más pequeñas que sus análogas americanas.
\end{abstract}

PALABRAS CLAVE: coastal-trapped disturbances; meteorología costera; tramontana; peligros aeronáuticos; HARMONIE-AROME.

\section{INTRODUCCIÓN}

Las coastal trapped disturbances (CTD) son corrientes de densidad que interaccionan con la orografía costera por el efecto de Coriolis y que se aceleran cerca de la costa (figura 1). Estos fenómenos han sido ampliamente estudiados (figura 2) en las costas de Estados Unidos (Mass y AlbRight, 1987), Sudáfrica (GILL, 1977) y Australia (REASON et al., 1999), y en menor medida en la costa cantábrica donde son denominadas «galernas» (ARASTI, 2001) y en las costas de Málaga donde se las ha denominado «corrientes de densidad atrapadas en la costa» (SÁnchez-LaulHé y Polvorinos, 1995). En este artículo se presenta en español la caracterización de este fenómeno en el Mediterráneo noroccidental descrito recientemente por GONZÁLEZ et al. (2018), y muestra por primera vez una visión integral de este fenómeno en la costa central catalana, en el que se incluye una climatología, un estudio mesoescalar del fenómeno mediante el uso de 
modelos de alta resolución (HARMONIE-AROME), un análisis de escala y de forzamientos y finalmente un estudio de sensibilidad del fenómeno al calentamiento diabático debido a la radiación solar.

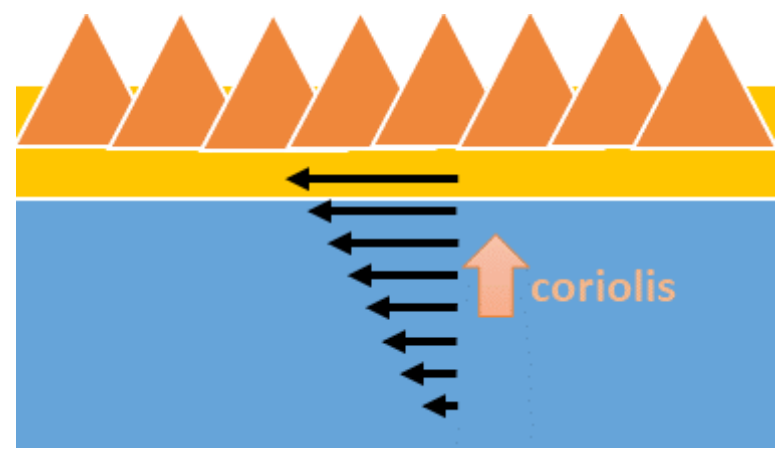

Figura 1. Esquema simplificado de las coastal trapped disturbances en el que el viento se acelera ageostróficamente cerca de las montañas.

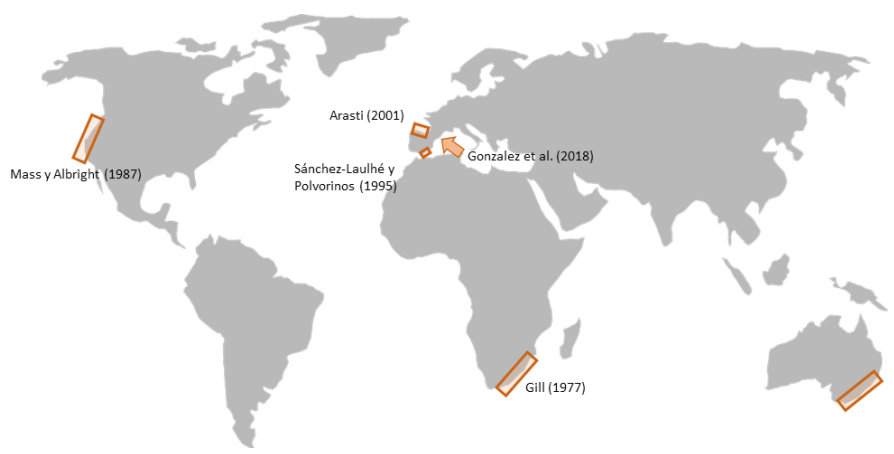

Figura 2. Distintas zonas del mundo donde las CTD han sido descritas.

\section{DESCRIPCIÓN DE LAS CTD}

Las CTD son un fenómeno de mesoescala que consiste en un incremento de velocidad y cambio de dirección bruscos del viento, junto con un aumento temporal de la cizalladura vertical y horizontal. Las conclusiones del estudio de GonZÁLEZ et al. (2018) indican que las CTD están asociadas comúnmente a las situaciones de tramontana-cierzo. Se producen en la frontera entre una masa de aire cálida y otra fría situada en la costa al norte de Barcelona que avanzan como una corriente de densidad hacia el suroeste en equilibrio antitríptico. El frente se desplaza en dirección hacia el aire cálido (al SW) debido a la diferencia de temperatura. La línea interactúa con la orografía debido a la fuerza de Coriolis que desvía ligeramente la masa de aire hacia la cordillera costero-catalana (figuras 3 y 4 ).

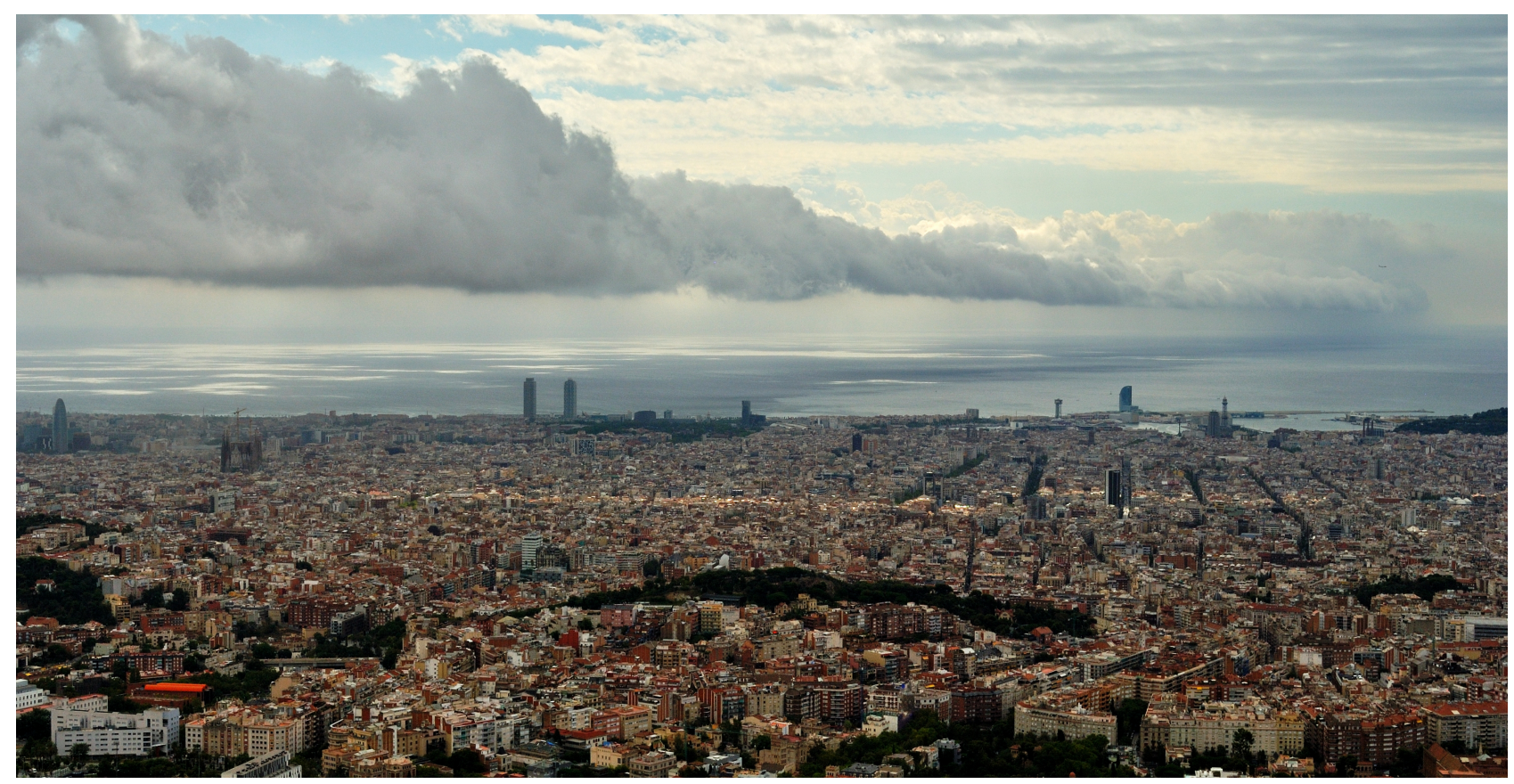

Figura 3. Fotografía de un Stratocumulus Volutus asociado a una CTD en Barcelona. Autor: Alfons Puertas. 


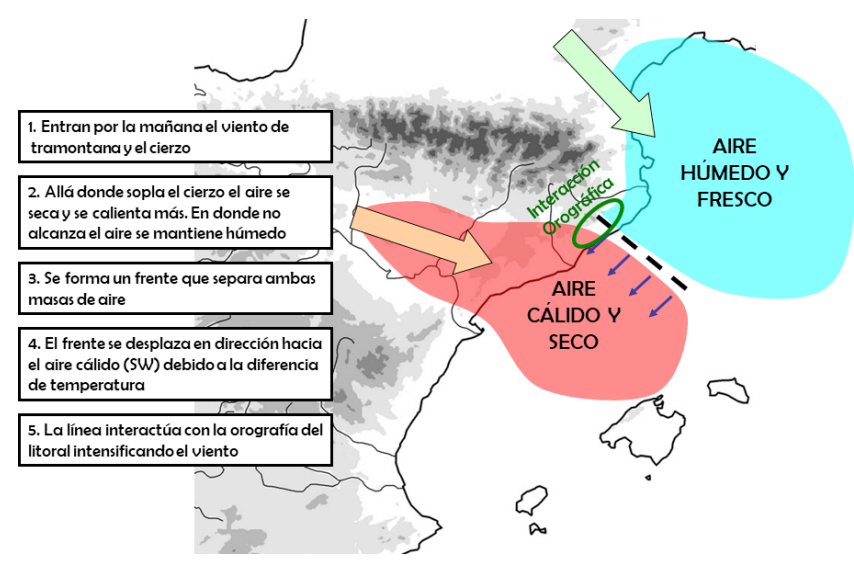

Figura 4. Modelo conceptual general de gran parte de las CTD en la costa central catalana.
Este fenómeno se da en masas de aire situadas en el nordeste de Cataluña, frías y estables, con un número de Froude de montaña cercano a 1 y un número de Burger mayor de 1. El origen del aire frío puede ser muy diverso; se han observado distintos orígenes, tanto dinámicos (la tramontana actuando como aire frío; aire frío empujado por una baja dinámica) como diabáticos (aire frío proviniendo de un cold pool; creación de un gradiente térmico por el calentamiento diabático del aire cálido).

\section{LAS CTD COMO PELIGRO AERONÁUTICO}

Este fenómeno afecta especialmente al aeropuerto de Barcelona debido a su situación geográfica cerca del delta del Llobregat. Al llegar a Barcelona esta línea produce una elevada cizalladura produciendo un peligro potencial para las operaciones aeronáuticas, en especial si el aeropuerto va a su máxima capacidad como han mostrado los estudios de González y Pascual (2013) en el caso del 12 de junio de 2012 o el de Méndez et al. (2018) para el caso del 10 de septiembre de 2017 (figura 5). Este fenómeno local constituye uno de los mayores peligros en las operaciones aéreas dentro del Estado español.

\section{SITUACIÓ LÍMIT AL PRAT}

\section{(ㅁ) "Tenim 45 avions al cel i no poden aterrar"}

- LAeroport del Prat va viure diumenge una situació crítica

Figura 5. Titular de la versión web de la noticia sobre el caos aéreo ocurrido el 10 de septiembre de 2017. El titular dice: «Tenemos 45 aviones en el cielo y no pueden aterrizar». Fuente: RAC1.

\section{CLIMATOLOGÍA DE LAS CTD}

El análisis de todos los eventos de CTD en Barcelona de 2010 a 2016 con rachas de por lo menos 14 m/s, indica que las CTD afectan un promedio de cuatro veces al año al aeropuerto de Barcelona. Estas CTD ocurren especialmente durante la época cálida del año y entre la tarde y la noche, lo que sugiere que el calentamiento diabático juega un papel importante (figura 6).

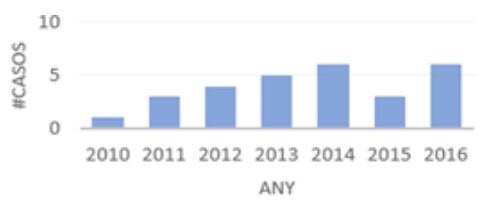

Distribución anual, mensual y horaria de los episodios de CTD en la costa catalana entre 2010 y 2016.
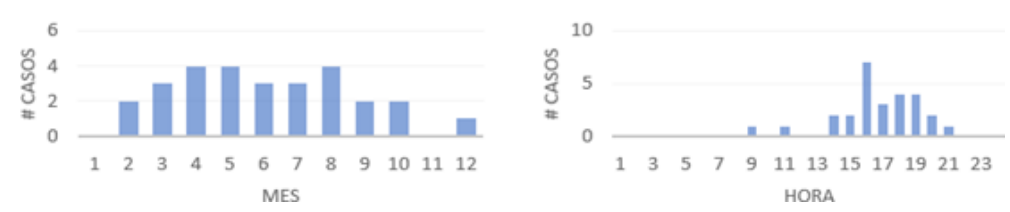


\section{CONCLUSIONES}

En esta comunicación se presenta un nuevo caso de coastal trapped disturbances (CTD) en la península ibérica. Este fenómeno es muy recurrente en los litorales españoles ya que ha sido previamente descrito en la costa cántabra (donde es conocido con el nombre de «galerna») y en las costas de Málaga. Además, se presentan los resultados del estudio de GonzÁLEZ et al. (2018) que ha realizado el primer estudio integral de este fenómeno a partir de simulaciones de alta resolución con el modelo HARMONIE-AROME. Con ello se ha podido observar que existe una gran diversidad de situaciones que pueden dar lugar a este fenómeno ya que el origen de la diferencia de temperaturas que da lugar a la corriente de densidad puede ser muy diverso.

HARMONIE-AROME es capaz de reproducir las CTD en ciertos casos. Al desarrollarse a escalas más pequeñas que sus homólogas americanas, su predecibilidad es más baja. Por ello, a partir de estas conclusiones de GonZÁlez et al. (2018) se propone desarrollar una herramienta operativa que pueda indicar las condiciones favorables para el desarrollo de este fenómeno en el aeropuerto de Barcelona. Esta herramienta debería incluir un análisis de escala, indicando cuándo el aire marítimo situado al norte de Barcelona presenta un ambiente adecuado. Con ello se podría mejorar la alerta temprana de este tipo de fenómenos y reducir el impacto en las operaciones aeroportuarias.

\section{AGRADECIMIENTOS}

Este trabajo se ha realizado con soporte parcial del grupo de investigación de la Generalitat de Catalunya ANTALP(Antarctic, Arctic and Alpine Environments, 2017-SGR-1102), el Institut de la Recerca de l'Aigua (IdRA) de la Universidad de Barcelona y los proyectos nacionales CGL201565627C32R (MINECO/ FEDER) y CGL201681828REDT (MINECO).

\section{REFERENCIAS}

Arasti, E., 2001. La Galerna Típica: Una perturbación atrapada en la costa. V Simposio Nacional de Predicción, Madrid.

GILL, A.E., 1977. Coastally trapped waves in the atmosphere. Quarterly Journal of the Royal Meteorological Society, 103, pp. 431-440.

González, S., Callado, A., Werner, E., Escribà, P., y Bech, J., 2018. CoastallyTrapped Disturbances Caused by the Tramontane Wind on the NorthWestern Mediterranean: Numerical Study and Sensitivity to ShortWave Radiation. Quarterly Journal of the Royal Meteorological Society, 144, 1321-1336. https:// doi.org/10.1002/qj.3320.

González, S. y Pascual, R., 2013. Strong winds of convective source in Barcelona on 12 June 2012. Tethys, 10, 13-23. https://doi.org/10.3369/tethys.2013.10.02.

MAss, C. F. y Albright, M. D., 1987. Coastal southerlies and alongshore of the west coast of North America: Evidence of mesoscale topographically trapped response to synoptic forcing. Monthly Weather Review, 115, 1707-1738. https://doi.org/10.1175/1520-0493(1987)115<1707:CSAASO>2.0.CO;2.

Méndez, A., Pons, M. R., Santos, I., Simarro, J., González, S., Pascual, R., Pladevall, A., Callado, A. y Compte, M., 2018. Episodio de cizalladura del 10-9-2017 en el aeropuerto de Barcelona. Sexto Simposio Nacional de Predicción de AEMET. Memorial Antonio Mestre.

RAC 1, 2017. 14 de setiembre. Situació límit al Prat. Rac1.cat. Recuperado de http://rac1.cat. 
Reason, C. J. C., Tory, K. J. y JAckson, P. I., 1999. Evolution of a southeast Australian coastally trapped disturbance. Meteorology and Atmospheric Physics, 70, 141-165. https://doi.org/10.1007/s007030050031.

SÁnchez-Laulhé, J. M, y Polvorinos, F., 1995. Entradas bruscas de vientos de levante en la costa norte de Alborán. Boletín de la Asociación Meteorológica Española, 18/19, 30-35. 
